nephrologists but also to professional nutritionists, dieticians, food manufacturers and anyone else working in the field of nutrition in renal disease in its widest sense.

This claim is indeed true, and the book makes stimulating reading and gives much practical clinical help as well as a concise and readable account of recent research and current theories of renal function in health and disease. It should prove useful both to specialist and non-specialist readers.

\section{Fertility and Contraception in the Human Female}

By John A. Loraine and E. Trevor Bell. Pp. 384 , illustrated. Edinburgh \& London: E. \& S. Livingstone, 1968. 55s.

A more appropriate title would be 'Hormone Excretion in Gynaecology with special reference to ovarian stimulation and contraception'. On this basis it is a first class book, packed with detailed and interesting information. For the progressive gynaecologist, particularly if he has a leaning towards endocrinology, the time required to read the book would be well spent.

It starts correctly but prosaically with the anatomy and physiology of ovulation. This is followed by a review of the best techniques for assessing hormone levels in urine and blood. The technical details of the assays are inadequate for the steroid chemist and boring for the clinician but they do indicate the amount of work involved in each estimation and the difficulty in providing accurate and reliable figures. With the description of hormone excretion patterns in the normal menstrual cycle and in various gynaecological disorders, the book comes alive, offering an authoritative collection of data, clarifying some problems and indicating lines for further study. The chapters on the selection of patients for treatment with clomiphene and gonadotrophin are clear and helpful. There is a competent general review of current opinion on oral contraception and a vivid final chapter on overpopulation.

Much of the information presented is based on the authors' own studies. As a result the text conveys both enthusiasm and conviction. Each chapter begins with an excellent and systematic index and ends with a comprehensive summary and bibliography. Altogether this is a book to read and then keep for reference.

\section{Recent Advances in Medicine. Fifteenth Edition}

Edited by D. N. Baron, Nigel Compston and A. M. Dawson. Pp. $x+423$, illustrated. London: J. \& A. Churchill, 1968. 65s. (hard back) 45s. (paper back).

Advance in medicine has been so rapid in the 4 years since the last edition of this book that a completely new range of subjects has been chosen and there has been therefore a complete change of authors. There are thirteen chapters each with a full list of references. Some, for example, 'Diseases of Muscle' are essentially reviews of many different clinical conditions; others, for example, 'Temperature Regulation and its Disorders', are texts of applied physiology. A broad outlook has led to the inclusion of chapters on Computers in Medicine and Environmental Factors in Disease. It is all most stimulating and rewarding reading of great general interest. The only disappointment is the chapter on Systemic Mycoses which includes little new material and gives an inadequate account of Aspergillosis, an important respiratory disease in Britain today. Perhaps this should have formed part of a chapter on immunological mechanisms in pulmonary disorders in which field so many important advances in understanding have recently been made. The book deserves to be widely read and bought, not only by medical libraries, but also individually by all who are interested in the study of medicine.
Logan Turner's Diseases of the Nose, Throat and Ear. Seventh Edition

Edited by John P. Stewart. Assisted by J. F. Birrell. Pp. 584. Bristol: John Wright, 1968. 70s.

As a newly qualified House Surgeon working in a busy ear, nose and throat Department of a London teaching Hospital 30 years ago, I purchased Logan Turner's textbook as my reference book. It had the advantage then, as now, of covering the whole field of oto-rhinolaryngology in one volume. I never regretted my choice and I still have that fourth edition. Comparing it with the latest seventh edition, now submitted for review, it can be seen that the subject matter has been brought completely up to date without greatly enlarging the volume. The best of the old illustrations have been retained, but many excellent photographs and diagrams illustrating modern techniques have been added. I would suggest, however, that the old illustration of myringotomy on page 427 might be changed in later editions as the commencement of the incision shown might well disrupt the incudo-stapedial joint. The coloured illustrations remain excellent.

There is a very good new chapter on laryngeal conditions in childhood.

The description of the classical operations for acute and chronic middle ear infections remain and, of course, microsurgical procedures for reconstruction of the middle ear and the treatment of otosclerosis are described in detail. This edition also includes the ultra sonic treatment of Meniere's syndrome. The useful appendix of formulae is still retained, but Chest Physicians would not approve of the laryngeal spray made up in Liquid Paraffin.

References are given at the end of each section. The large number of articles quoted from Journals published since the 1961 , sixth edition, is an indication of the amount of new material in this volume. The print and paper remain excellent and the index comprehensive.

I still consider Logan Turner's Diseases of the Nose, Throat and Ear the ideal textbook for House Surgeons commencing a career in otolaryngology.

Fundamental Techniques of Plastic Surgery. Fourth Edition By IAN A. MCGregor. Pp. 298. Edinburgh \& London: E. \& S. Livingstone, 1968. 35s.

This book is already well known, and the new edition, the fourth in 8 years, confirms its importance. As before, the first section describes techniques used in plastic surgery, and the second deals with their application in other fields. There has been much re-writing, and a slight reduction in size. Advice is given clearly and uncompromisingly on a wide range of subjects, and suggestions for treatment are invariably sound and reliable. The new discussion of combined injuries of skin and bone is specially good. After each chapter there is a small but useful bibliography. Illustrations are profuse and helpful, and even the photographs are clear.

The work will continue to be a textbook for trainees in plastic surgery, and a valuable reference book where a plastic opinion is not available.

Some Diseases of Animals Communicable to Man in Britain Proceedings of a Symposium organized by The British Veterinary Association and The British Small Animal Veterinary Association, London 1966. Edited by Oliver Graham-Jones. Pp. xvi +338 , illustrated. Oxford: Pergamon Press, 1968. 126s.

This publication of the proceedings of a symposium held in 1966 although not claiming to be comprehensive covers a 